\title{
Improved A* Algorithm For Time-dependent Vehicle Routing Problem
}

\author{
Zhang Yi \\ college of aerospace and material engineering, national \\ university of defense technology, \\ changsha 410073 , china
}

\begin{abstract}
Time-dependent vehicle routing problem (TDVRP) is concerned with vehicle routing optimization in road networks with fluctuant link travel time. Firstly, Time-dependent functions about routing time indicator and routing threat indicator are introduced. Secondly, a time-dependent heuristic function including routing distance indicator, routing time indicator and routing threat indicator is constructed, and an improved $a^{*}$ algorithm is promoted for tdvrp. Finally, the algorithm is validated by using simulations.
\end{abstract}

Keywords-Time-dependent vehicle routing Problem, Heuristic algorithm, Threat, Time-dependent heuristic function

\section{INTRODUCTION}

Vehicle routing problem (VRP) was firstly proposed by Dantzig in 1959[1]. For simplicity, most researches are concentrated on static networks. Actually, the travel speed and times are time-dependent due to traffic status and weather conditions. Furthermore, Exposure threats of vehicles/teams are different owing to periodic motion of satellite. Thus, it's a kind of TDVRP actually, which implies that the costs of two nodes not only depend on distances, but also departure time[2].

Algorithms for solving TDVRP can be classified into two categories: precise algorithm and heuristic algorithm. The former includes: Branch and bound algorithm[3], dynamic programming algorithm[4,5] and tree search algorithm[6], etc. TDVRP is a NP-hardness, and the precise solution can be obtained when the problem is simple. When the problem gets complicated, the computing time and storage space will experience rapid growth, and the precise algorithm will be invalidate. Therefore, heuristic algorithm is adapted by most researchers, which involves greedy algorithm[7], tabu search algorithm[8], ant colony algorithm $[9,10]$ and genetic algorithm[11], etc.

$A^{*}$ is a kind of heuristic algorithm, and it was adopted by Vachtevanos[12] and Szczerba[13] for best track searching, where the cost function was represented by the weighted constraints of distance, threat and mobility. So far, researches on TDVRP by using $\mathrm{A}^{*}$ algorithm is mere in the literature.

In the paper, improved $A^{*}$ algorithm is proposed for TDVRP. Step function and piecewise continuous function are introduced to represent time-dependent travel speeds and exposure threats. The heuristic algorithm of improved $\mathrm{A}^{*}$ is constructed by mileage indicator function, time indicator function and threat indicator function. At last, the algorithm is validated by using simulations.

\author{
Tang guojin, Chen Lei \\ college of aerospace and material engineering, national \\ university of defense technology, \\ changsha 410073 , china
}

\section{TIME-DEPENDENT VEHICLE ROUTING PROBLEM}

\section{A. Mathematical model}

$R(N, D, V, S)$ reprents the time-dependent network map, where $N=\left\{n_{1}, n_{2}, \cdots, n_{N}\right\}$ is the set of all nodes, $D=\left\{d_{k}(i, j) \mid i, j=1,2, \cdots, N, i \neq j, k=1,2, \cdots, M\right\}$ is the set of all sections, $\quad V(t)=\left\{v_{k}(t) \mid k=1,2, \cdots, M\right\} \quad$ and $S(t)=\left\{s_{k}(t) \mid k=1,2, \cdots, M\right\}$ are the sets of timedependent travel speed and threat, respectively. The weights of section $d_{k}(i, j)$ are $F_{D k}, F_{T k}\left(t_{m}\right)$ and $F_{S k}\left(t_{m}\right)$, where $F_{D k}$ is the length of $d_{k}, F_{T k}\left(t_{m}\right)$ and $F_{S k}\left(t_{m}\right)$ are the travel time and threat when passing through $d_{k}: n_{i} \rightarrow n_{j}$ at departure time $t_{m}$.

The time-dependent optimal path can be described as follows: we try to find a path $\prod_{n_{0} n_{d}}\left(t_{0}\right)=\left\{\left(n_{0}, t_{0}\right), \cdots,\left(n_{i}, t_{i}\right), \cdots,\left(n_{d}, t_{d}\right)\right\} \quad$ in $R(N, D, V, S)$ to minimize the cost function $F\left(t_{0}\right)=\sum_{j} \sum_{i=D, T, S} \lambda_{i} F_{i j}$, whose departure time, starting node and end node are $t_{0}, n_{0}$ and $n_{d}$, respectively.

\section{B. Evaluating indicators of optimal path}

The mileage indicator, time indicator and threat indicator are used to construct evaluating indicators of vehicle support in troop transportation. The indicators are described as follows:

1) Mileage indicator

Mileage indicator is the sum of traveled path length

$$
F_{D}=\sum_{i=1}^{N} F_{D i}
$$

where, $F_{D i}$ is the length of the $i$ th path.

2) Time indicator

Time indicator is the total time passing though all

$$
\begin{aligned}
& \text { the paths } \\
& F_{T}=\sum_{i=1}^{N} F_{T i}
\end{aligned}
$$

where, $F_{T i}$ is the time when passing though the $i$ th path. 


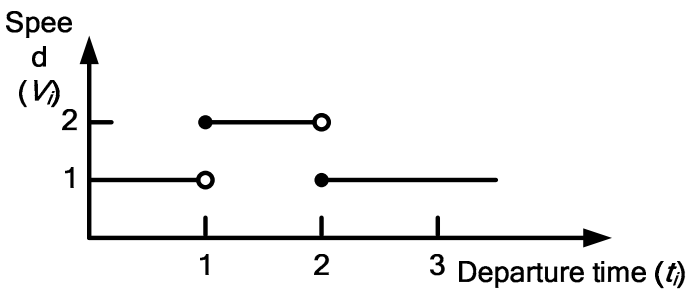

Figure 1 Diagram of time-dependent speed

Under traffic conditions, the permissible speed of the $i$ th path is time-dependent, as shown in Figure 1.

Travel time $F_{T i}$ and path length $F_{D i}$ meet the following relations

$$
F_{D i}=\int_{t_{i 0}}^{t_{i 0}+F_{T i}} V_{i}(t) d t
$$

where, $F_{D i}$ is the length of the $i$ th path, $t_{i 0}$ is the initial time reaching the $i$ th path, $F_{T i}$ is the travel time on the $i$ th path, $V_{i}(t)$ is the Time-varying speed on the $i$ th path.

\section{3) Threat indicator}

Threat indicator is a measure of threat when passing through all paths, which can be expressed as follows

$$
F_{S}=\sum_{i=1}^{N} F_{S i}
$$

where, $F_{S i}$ is the threat when passing through the $i$ th path.

The threat degree $F_{T i}$ and its distributing function $f_{s i}(t)$ meet the following relations

$$
F_{S i}=\int_{t_{i 0}}^{t_{i 0}+F_{T i}} f_{s i}(t) d t
$$

where, $t_{i 0}$ is the initial time reaching the $i$ th path, $F_{T i}$ is the travel time on the $i$ th path, $f_{s i}(t)$ is the distributing threat function of the $i$ th path.

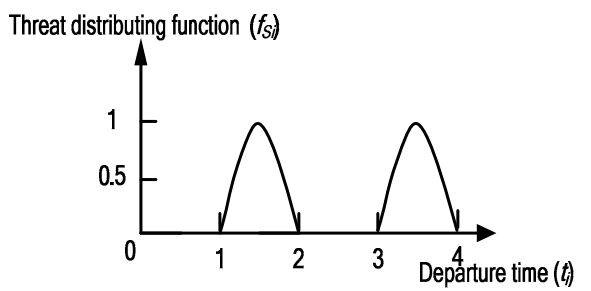

Figure 2 Diagram of distributing threat function

It should be noted that, as time indicator $F_{T}$ and threat indicator $F_{S}$ are functions of departure time $T_{0}$, the optimal paths are different according to different departure time windows.

\section{A* ALGORITHM FOR TIME-DEPENDENT VEHICLE} ROUTING PROBLEM

\section{A. A* algorithm}

A * algorithm is a heuristic search algorithm in artificial intelligence. Peter Hart, Nils Nilsson and Bertram Raphael first described the algorithm in 1968. It is an extension of Edsger Dijkstra's 1959 algorithm. A* achieves better performance (with respect to time) by using heuristics. Noted for its performance and accuracy, it enjoys widespread use.

It uses a distance-plus-cost heuristic function to determine the order in which the search visits nodes in the tree. The distance-plus-cost heuristic is a sum of two functions: the path-cost function, which is the cost from the starting node to the current node; the admissible function, which is the "heuristic estimate" of the distance to the goal.

A distance-plus-cost heuristic function has the following expression

$$
f^{*}(i)=g(i)+h^{*}(i)
$$

where, $g(i)$ is the path-cost function, $h^{*}(i)$ is the admissible function.

\section{B. Improved $A *$ algorithm}

The father node information is involved in the improved A* algorithm. The distance-plus-cost heuristic function can be written as

$$
f^{*}(i)=g(i)+h^{*}(i)+h^{*}(j)
$$

where, $g(i)$ and $h^{*}(i)$ share the same definition as in Eq.(6). $h^{*}(j)$ is the "heuristic estimate" of the distance from father node to the goal.

The improved $\mathrm{A}^{*}$ algorithm makes the algorithm search directions tend to the goal faster, and significantly reduces the number of experienced nodes ${ }^{[11,12]}$.

\section{Heuristic function}

A time-varying heuristic function is constructed from Eq.(7) as following

$$
\begin{aligned}
F\left(i, T_{0}\right) & =\lambda_{D}\left[F_{D}(i)+F_{D E}(i)+F_{D E}(j)\right]+\lambda_{T}\left[F_{T}\left(i, T_{0}\right)+F_{I E}(i)+F_{I E}(j)\right] \\
& +\lambda_{S}\left[F_{S}\left(i, T_{0}\right)+F_{S E}(i)+F_{S E}(j)\right]
\end{aligned}
$$

where, $F_{D}$ is mileage indicator, $F_{T}$ is time indicator,

$F_{S}$ is threat indicator, $\lambda_{D}, \lambda_{T}$ and $\lambda_{S}$ are weighting coefficients of mileage, time and threat, respectively. $T_{0}$ is the departure time, $F_{D E}(i)$ and $F_{D E}(j)$ are Manhattan distances to the destination from the current node and father node, respectively. $F_{T E}(i)$ and $F_{S E}(i)$ are admissible functions from current node to the destination, $F_{T E}(j)$ and 
$F_{S E}(j)$ are admissible functions from father node to the destination.

$$
\begin{gathered}
F_{D E}(m)=\lambda_{d}\left(\left|x_{m}-x_{d}\right|+\left|y_{m}-y_{d}\right|\right)(9) \\
F_{T E}(m)=\lambda_{t} \frac{F_{T}\left(m, T_{0}\right)}{F_{D}(m)} F_{D E}(m)(10) \\
F_{S E}(m)=\lambda_{s} \frac{F_{S}\left(m, T_{0}\right)}{F_{D}(m)} F_{D E}(m)(11)
\end{gathered}
$$

where, $m=i, j, \lambda_{d}, \lambda_{t}$ and $\lambda_{s}$ are constant weighting coefficients.

\section{IV. . Simulations}

Consider a road network of vehicle support in troop transportation, which involves 100 nodes and 190 roads. The improved $\mathrm{A}^{*}$ algorithm is adopted to find an optimal path. Simulation parameters are $\lambda_{d}=1, \lambda_{t}=1.2, \lambda_{s}=0.4, T_{0}=0$, $\lambda_{D}=3.5, \lambda_{T}=1, \lambda_{S}=1$ 。

Simulation results of different optimization schemes are shown in Tab.1. It can be seen that, the costs of integrated optimization scheme are the compromise of single indicator optimization scheme. In practice, the interested indicator can be stressed by adjusting the corresponding weights.

As shown in Tab.2, costs of integrated optimization scheme depend on departure times. Because the travel speed and threat distributing function are time-varying. When $T_{0}=0$, the integrated costs is minimum. But, the optimal departure time will be different when the time-dependent functions changes.

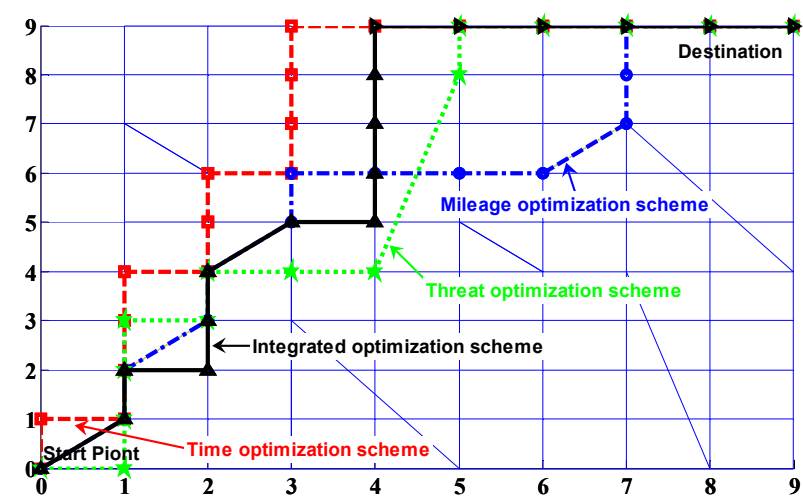

Figure 3 The optimal paths of different schemes

The optimal paths of different schemes at $T_{0}=0$ is shown in Figure 3, it can be seen that, the optimal paths are deferent when different optimization schemes are adopted.

\section{CONCLUSIONS}

The improved $\mathrm{A}^{*}$ algorithm is promoted for TDVRP in the paper. The travel speed is represented by step functions, the threat of satellites is represented by using piecewise continuous function. A time-dependent heuristic function is constructed involving mileage indicator, time indicator and threat indicator. Furthermore, the farther information is included in the improved $A^{*}$ algorithm to improve search efficiency. Simulation results show the effectiveness of the promoted algorithm.

\section{REFERENCES}

[1] Dantzig G B, Ramser J H. The truck dispatching problem [J]. Management Science, 1959, 6(1),PP:80-91.

[2] Beasley J. Adapting the savings algorithm for varying inter-customer travel times [J]. Omega International Journal of Management Science, 1981, 9(6),PP:685-659.

[3] Fox K.R. Production scheduling on parallel lines with dependencies[D]. Doctor Degree Dissertation, Johns Hopkins University. 1973.

[4] Balinsk M, Quandi R. On an integer program for a delivery problem[J]. Operations Research. 1964, 12(2), PP:300-304.

[5] Fischetti M, Laporte G, Martello S. The delivery man problem and cumulative metroids[J]. ORSA Journal on Computing. 1990, 2(4), PP:353-364

[6] Bianco L, Mingozzi A, Ricciardelli S. The traveling salesman problem with cumulative cost[J]. Networks. 1993,23(2), PP:81-91.

[7] Malandraki C Daskin MS. Time dependent vehicle routing problems, PP: formulations properties and heuristic algorithms[J]. Transportation Science. 1992, 26, PP:185-200.

[8] Ichoua S, Gendreau M, Potvin JY. Vehicle dispatching with timedependent travel times[J]. European Journal of Operational Research. 2003, 144(2), PP:379-396.

[9] Donati A V, Gambardella L M, Casagrande N, et al. Time dependent vehicle routing problem with an ant colony system[A]. Istituto Dalle Molle di Studisull Intelligenza Artificiale (IDSIA) Galleria 2, 6928 Manno, Switzerland, 2003.

[10] Donati AV, Montemanni R, Casagrande N, Rizzoli AE, Gambardella LM. Time dependent vehicle routing problem with a multi ant colony system[J]. European Journal of Operational Research. 2008, 185(3), PP:1174-1191.

[11] Haghani A, Jung S. A dynamic vehicle routing problem with timedependent travel tiems[J]. Computer \& Operations Research. 2005, 32(11), PP:2959-2986.

[12] G.. Vachtevanos et al. Autonomous vehicles: from flight control to mission planning using fuzzy logic techniques[J]. Proceedings of the 13th international Digital Signal Processing Conference, 1997. PP:977-981.

[13] Robert J.Szczerba et al. Robust algorithm for real-time route planning $[\mathrm{J}]$. IEEE Transactions on Aerospace and Electronic Systems ,Vol.36 No.3,2000. PP:869-878. 
TABLE I. COSTS OF DIFFERENT SCHEMES

\begin{tabular}{cccc}
\hline Optimization schemes & Mileage costs & $\begin{array}{c}\text { Time } \\
\text { costs }\end{array}$ & $\begin{array}{c}\text { Threat } \\
\text { costs }\end{array}$ \\
\hline Optimal mileage & $\mathbf{1 5 . 6 6}$ & 24.52 & 12.15 \\
Optimal time & 18.00 & $\mathbf{2 0 . 9 3}$ & 8.82 \\
Optimal threat & 17.12 & 20.24 & $\mathbf{6 . 9 5}$ \\
Integrated Optimization & $\mathbf{1 6 . 8 3}$ & $\mathbf{2 2 . 8 7}$ & $\mathbf{8 . 9 3}$ \\
\hline
\end{tabular}

TABLE II. COSTS OF INTEGRATED OPTIMIZATION SCHEME AT DIFFERENT DEPARTURE TIMES

\begin{tabular}{|c|c|c|c|c|}
\hline $\begin{array}{l}\text { Departu } \\
\text { re } \\
\text { time } \\
\left(T_{0}\right)\end{array}$ & $\begin{array}{l}\text { Milea } \\
\text { ge } \\
\text { costs }\end{array}$ & $\begin{array}{c}\text { Tim } \\
\text { e } \\
\text { costs }\end{array}$ & $\begin{array}{l}\text { Threa } \\
\mathrm{t} \\
\text { costs }\end{array}$ & $\begin{array}{c}\text { Integrate } \\
\mathrm{d} \\
\text { costs }\end{array}$ \\
\hline $\mathbf{0}$ & 16.83 & 22.87 & 8.93 & 90.70 \\
\hline 0.5 & 16.83 & $\begin{array}{c}23 \\
60\end{array}$ & 8.71 & 91.20 \\
\hline 1 & 17.41 & $\begin{array}{c}23 \\
45\end{array}$ & 7.39 & 91.79 \\
\hline 1.5 & 17.41 & $\begin{array}{c}23 \\
45\end{array}$ & 7.14 & 91.55 \\
\hline 2 & 17.41 & $\begin{array}{c}23 \\
45\end{array}$ & 7.05 & 91.46 \\
\hline 2.5 & 17.41 & $\begin{array}{l}23 . \\
81\end{array}$ & 7.08 & 91.84 \\
\hline 3 & 16.83 & $\begin{array}{l}25 . \\
87\end{array}$ & 8.93 & 93.70 \\
\hline
\end{tabular}

